\title{
COHEN-MACAULAY, GORENSTEIN, COMPLETE INTERSECTION AND REGULAR DEFECT FOR THE TENSOR PRODUCT OF ALGEBRAS
}

\author{
S. BOUCHIBA ${ }^{(\star)}$, J. CONDE-LAGO, AND J. MAJADAS
}

\begin{abstract}
Aвstract. This paper main goal is to measure the defect of Cohen-Macaulayness, Gorensteiness, complete intersection and regularity for the tensor product of algebras over a ring. For this sake, we determine the homological invariants which are inherent to these notions, such as the Krull dimension, depth, injective dimension, type and embedding dimension of the tensor product constructions in terms of those of their components. Our results allow to generalize various theorems in this topic especially [BK1. Theorem 2.1], [TY] Theorem 6] and [M] Theorems 1 and 2] as well as two Grothendieck's theorems on the transfer of Cohen-Macaulayness and regularity to tensor products over a field issued from finite field extensions. To prove our theorems on the defect of complete intersection and regularity, the homology theory introduced by André and Quillen for commutative rings turns out to be an adequate and efficient tool in this respect.
\end{abstract}

\section{INTRODUCTION}

All rings of this paper are assumed to be unitary and Noetherian and all ring homomorphisms are unital. In particular, the tensor product $A \otimes_{R} B$ of two Noetherian algebras $A$ and $B$ over a Noetherian ring $R$ is supposed to be Noetherian.

In [BK1], the authors were concerned with the problem of the transfer of the Cohen-Macaulayness property to the tensor product of algebras over a field. Its main theorem proves that the tensor product $A \otimes_{k} B$ of two algebras $A$ and $B$ over a field $k$, assuming Noetherianity of $A \otimes_{k} B$, is Cohen-Macaulay if and only if so are $A$ and $B$. This theorem extends a result of Grothendieck in this regard when the components of the considered tensor product are two field extensions one of which is finitely generated over $k$. The transfer of the Gorenstein and complete intersection properties to these tensor product constructions was investigated by Watanabe, Ishikawa, Tachibana and Otsuka in [WITO] and subsequently by Tousi and Yassemi in [TY], proving similar theorems for these two notions as the above one on Cohen-Macaulayness.

The regularity property still resists to any effort of improvement at the level enjoyed by the above three notions. Indeed, contrary to the above notions, a Noetherian tensor product of two field extensions of a field $k$ is not regular in general. In 1965 , Grothendieck proved that $K \otimes_{k} L$ is a regular ring provided $K$ or $L$ is a finitely generated separable extension field of $k$ [EGA IV], Seconde Partie, Lemma 6.7.4.1] and the finiteness hypotheses were dropped in [Sh1, Note]. In 1969, Watanabe,

Date: February 13, 2018.

2010 Mathematics Subject Classification. 13H10, 13C15, 13H05, 13C14, 13 D03.

Key words and phrases. Tensor product of algebras, Cohen-Macaulay ring, Gorenstein ring, complete intersection ring, regular ring, Krull dimension, depth, embedding dimension.

${ }^{\star}{ }^{\star}$ Supported by KFUPM under DSR Research Grant \# RG1212. 
Ishikawa, Tachibana, and Otsuka, showed that under a suitable condition tensor products of regular rings are complete intersections [WITO, Theorem 2, p. 417] and subsequently Tousi and Yassemi proved that a Noetherian tensor product of two $k$-algebras $A$ and $B$ is regular if and only if so are $A$ and $B$ in the special case where $k$ is perfect. In this context, the main theorem of [BK2] establishes necessary and sufficient conditions for a Noetherian tensor product of two extension fields of $k$ to inherit regularity and hence generalizes Grothendieck's aforementioned result. Recently, a more general result (over a base ring) was obtained in [M].

In the stream of the above achievements, we aim in this paper to go farther in this direction by measuring the defect of Cohen-Macaulayness, Gorensteiness, complete intersection and regularity for the tensor product of algebras over a ring $R$. To this purpose, it is clearly essential to determine the very invariants which are inherent to these notions such as the Krull dimension, depth, injective dimension, type and embedding dimension of the tensor product constructions in terms of those of their components. That is our objective in the most part of this paper which allows us to evaluate the defect of the above notions for the tensor products under some flatness condition. Our results allow to generalize the above theorems in [BK1], [BK2], [M], [TY], [WITO]. In the last two sections of the paper we use the homology theory of André and Quillen. For the convenience of the reader, we include a whole section to give a brief introduction to such homology, including the results used in this paper.

\section{Acknowledgement}

We are indebted to $S$. Kabbaj for his many contributions to this paper.

\section{Cohen-Macaulay Rings}

This section main goal is to determine the Cohen-Macaulayness defect of the tensor product $A \otimes_{R} B$ of algebras $A$ and $B$ over an arbitrary ring $R$ in local and global cases. Also, we generalize [BK1, Theorem 2.1] which characterizes the Cohen-Macaulayness of the tensor product $A \otimes_{k} B$ of algebras $A$ and $B$ over a field $k$ in terms of the Cohen-Macaulayness of $A$ and $B$.

Let $R$ be a ring and let $A$ and $B$ be $R$-algebras. First, it is worth noting that the tensor product $A \otimes_{R} B$ over $R$ might be trivial even if $A$ and $B$ are not so. Of course, the interesting case is when $A \otimes_{R} B \neq\{0\}$ which makes it legitimate to seek conditions on the underlying components $R, A$ and $B$ of the tensor product which guarantee $A \otimes_{R} B \neq\{0\}$. That is the purpose of our first result.

For a given $R$-algebra $A$, denote by $f_{A}: R \longrightarrow A$, with $f_{A}(r)=r \cdot 1_{A}$ for any $r \in R$ and where $1_{A}$ is the unit element of $A$, the ring homomorphism defining the structure of algebra of $A$ over $R$. Also, if $A$ and $B$ are $R$-algebras, we denote by $\mu_{A}: A \longrightarrow A \otimes_{R} B$ and $\mu_{B}: B \longrightarrow A \otimes_{R} B$ the canonical algebra homomorphisms over $A$ and $B$, respectively, such that $\mu_{A}(a)=a \otimes_{R} 1$ and $\mu_{B}(b)=1 \otimes_{R} b$ for each $a \in A$ and each $b \in B$. Observe that the following diagram is commutative: 


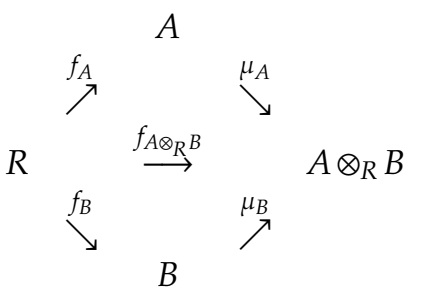

Throughout this section, given ideals $I, J$ and $H$ of $A, B$ and $A \otimes_{R} B$, respectively, we adopt the following notation for easiness: $I \cap R:=f_{A}^{-1}(I), J \cap R:=f_{B}^{-1}(J)$ and $H \cap A:=\mu_{A}^{-1}(H), H \cap B:=\mu_{B}^{-1}(H)$. By commutativity of the above diagram $(H \cap$ $A) \cap R=(H \cap B) \cap R$. Also, given an ideal $J$ of $B$, we denote by $A \otimes_{R} J$ the ideal $\mu_{B}(J)\left(A \otimes_{R} B\right)$ of $A \otimes_{R} B$ generated by $\mu_{B}(J):=\left\{1 \otimes_{R} b: b \in J\right\}$.

We begin by characterizing when the tensor product $A \otimes_{R} B$ of two algebras $A$ and $B$ over a ring $R$ is not trivial. Also, we characterize when, given two prime ideals $I$ and $J$ of $A$ and $B$, respectively, there exists a prime ideal $P$ of $A \otimes_{R} B$ such that $P \cap A=I$ and $P \cap B=J$.

Proposition 2.1. Let $R$ be a ring and $A, B$ be two R-algebras. Then

(1) $A \otimes_{R} B \neq\{0\}$ if and only if there exists a prime ideal I of $A$ and a prime ideal $J$ of $B$ such that $I \cap R=J \cap R$.

(2) Let I be a prime ideal of $A$ and $J$ be a prime ideal of $B$. There exists a prime ideal $P$ of $A \otimes_{R} B$ such that $P \cap A=I$ and $P \cap B=J$ if and only if $I \cap R=J \cap R$.

Proof. (2) See [EGA I, Corollaire 3.2.7.1.(i)].

(1) Since $A \otimes_{R} B \neq\{0\}$ if and only if $\operatorname{Spec}\left(A \otimes_{R} B\right) \neq \emptyset$, it follows from (2).

We denote by $\mathcal{R}$ the class of Noetherian local rings $(S, m, k)$, where $m$ is the maximal ideal of $S$ and $k$ its residue field. Let $\Lambda$ consist of the functions

$$
\lambda: \mathcal{R} \longrightarrow \mathbb{N} \cup\{+\infty\}
$$

vanishing on any field and satisfying following property: for any flat local homomorphism $(S, m, k) \longrightarrow(T, n, l)$, we have $\lambda(T)=\lambda(S)+\lambda\left(\frac{T}{m T}\right)$. Since from the identity map of a field $k$ we obtain $\lambda(k)=\lambda(k)+\lambda(k)$, the condition that $\lambda$ vanishes on field $k$ is equivalent to $\lambda(k) \neq+\infty$. The sum of two such functions is also an element of $\Lambda$ as well as the difference (provided it is defined and takes values in $\mathbb{N} \cup\{+\infty\}$ ).

The following invariants are well known examples of elements of $\Lambda$.

Proposition 2.2. The following functions from $\mathcal{R}$ to $\mathbb{N} \cup\{+\infty\}$ are elements of $\Lambda$ :

(1) The Krull dimension (denoted by dim).

(2) The depth (denoted by depth).

(3) The self-injective dimension (denoted by id).

(4) The codepth (denoted by codepth).

(5) The complete intersection defect (denoted by $\mathrm{d}$ ).

Proof. (1) See [Mt, Theorem 15.1].

(2) See [Mt, Corollary, p. 181] or [EGA IV, Seconde Partie, Proposition 6.3.1].

(3) See [FT, Corollary 1].

(4) Since codepth $=$ dim - depth, then (4) follows from (1) and (2).

(5) See [Av, Proposition 3.6]. 
Let $\Lambda^{\prime}$ be the class of functions $\lambda^{\prime}: \mathcal{R} \longrightarrow \mathbb{N} \cup\{+\infty\}$ verifying

$$
\lambda^{\prime}\left(R_{n}\right)+\lambda^{\prime}\left(\left(A \otimes_{R} B\right)_{P}\right)=\lambda^{\prime}\left(A_{p}\right)+\lambda^{\prime}\left(B_{q}\right)+\lambda^{\prime}\left(\left(k_{A}(p) \otimes_{k_{R}(n)} k_{B}(q)\right)_{P\left(k_{A}(p) \otimes_{k_{R}(n)} k_{B}(q)\right)}\right)
$$

and vanishing on any field (equivalently taking finite values on any field), where $A$ and $B$ are $R$-algebras and $P \in \operatorname{Spec}\left(A \otimes_{R} B\right)$ with $p:=P \cap A, q:=P \cap B$ and $n:=P \cap R$, and either $A_{p}$ or $B_{q}$ is flat over $R_{n}$.

We are going to see that $\Lambda=\Lambda^{\prime}$.

Theorem 2.3. Let $A$ and $B$ be two R-algebras. Let $P \in \operatorname{Spec}\left(A \otimes_{R} B\right)$ with $p:=P \cap A$, $q:=P \cap B$ and $n:=P \cap R$. Assume that $A_{p}$ is flat over $R_{n}$. Then, for any $\lambda \in \Lambda$,

$$
\lambda\left(\left(A \otimes_{R} B\right)_{P}\right)=\lambda\left(\frac{A_{p}}{n A_{p}}\right)+\lambda\left(B_{q}\right)+\lambda\left(\left(k_{A}(p) \otimes_{k_{R}(n)} k_{B}(q)\right)_{P\left(k_{A}(p) \otimes_{k_{R}(n)} k_{B}(q)\right)}\right) .
$$

The proof of Theorem 2.3 requires the following preparatory lemma.

Lemma 2.4. Let $R$ be a ring. Let $A$ and $B$ be $R$-algebras.

(1) Let $J$ be an ideal of $B$ such that $J \cap R:=m$ is a maximal ideal of $R$. Then

$$
\frac{A \otimes_{R} B}{A \otimes_{R} J} \cong \frac{A}{m A} \otimes_{k} \frac{B}{J} \cong A \otimes_{R} \frac{B}{J}
$$

where $k$ denotes the field $\frac{R}{m}$.

(2) Let $P$ be a prime ideal of $A \otimes_{R} B$. Let $p:=P \cap A, q:=P \cap B$ and $n:=P \cap R$. Then

$$
\frac{\left(A \otimes_{R} B\right)_{P}}{\left(A \otimes_{R} q\right)_{P}} \cong\left(\frac{A_{p}}{n A_{p}} \otimes_{k_{R}(n)} k_{B}(q)\right)_{P\left(\frac{A_{p}}{n A_{p}} \otimes_{k_{R}}(n) k_{B}(q)\right)} .
$$

Proof. (1) Since $A \otimes_{R} J=m A \otimes_{R} B+A \otimes_{R} J$ because $m A \otimes_{R} B \subset A \otimes_{R} J$, using the exact sequences

$$
\begin{gathered}
m A \longrightarrow A \longrightarrow A / m A \longrightarrow 0 \\
J \longrightarrow B \longrightarrow B / J \longrightarrow 0
\end{gathered}
$$

it follows from [Bo, Chapter II, §3.6, Proposition 6] that

$$
\frac{A \otimes_{R} B}{A \otimes_{R} J} \cong \frac{A \otimes_{R} B}{m A \otimes_{R} B+A \otimes_{R} J} \cong \frac{A}{m A} \otimes_{R} \frac{B}{J} \cong\left(\frac{A}{m A} \otimes_{R} k\right) \otimes_{k} \frac{B}{J} \cong \frac{A}{m A} \otimes_{k} \frac{B}{J}
$$

(2) First, note that $\left(A \otimes_{R} B\right)_{P} \cong\left(A_{p} \otimes_{R_{n}} B_{q}\right)_{P\left(A_{p} \otimes_{R_{n}} B_{q}\right)}$. Then

$$
\frac{\left(A \otimes_{R} B\right)_{P}}{\left(A \otimes_{R} q\right)_{P}} \cong \frac{\left(A_{p} \otimes_{R_{n}} B_{q}\right)_{P\left(A_{p} \otimes_{R_{n}} B_{q}\right)}}{\left(A_{p} \otimes_{R_{n}} q B_{q}\right)_{P\left(A_{p} \otimes_{R_{n}} B_{q}\right)}} \cong\left(\frac{A_{p} \otimes_{R_{n}} B_{q}}{A_{p} \otimes_{R_{n}} q B_{q}}\right)_{\frac{P\left(A_{p} \otimes_{R_{n}} B_{q}\right)}{A_{p} \otimes_{R_{n}} q B_{q}}} .
$$

Now, as $q B_{q} \cap R_{n}=n R_{n}$ is maximal in $R_{n}$, we get the desired isomorphism applying (1).

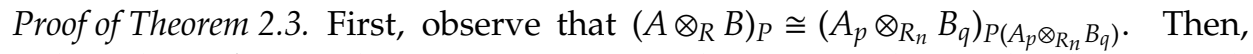
without loss of generality, we may assume that $R, A$ and $B$ are local rings with maximal ideals $n, p$ and $q$, respectively, such that $A$ is flat over $R, P\left(A \otimes_{R} B\right)_{P} \cap A=p$, $P\left(A \otimes_{R} B\right)_{P} \cap B=q$ and $P\left(A \otimes_{R} B\right)_{P} \cap R=p \cap R=q \cap R=n$. As the ring homomorphism 
$R \rightarrow A$ is flat, we get $B \rightarrow A \otimes_{R} B$ is a flat homomorphism which induces the flat homomorphism of rings $B \rightarrow\left(A \otimes_{R} B\right)_{P}$. Let $\lambda \in \Lambda$. Then, by hypothesis,

$$
\begin{aligned}
\lambda\left(\left(A \otimes_{R} B\right)_{P}\right) & =\lambda(B)+\lambda\left(\frac{\left(A \otimes_{R} B\right)_{P}}{q\left(A \otimes_{R} B\right)_{P}}\right) \\
& =\lambda(B)+\lambda\left(\left(\frac{A \otimes_{R} B}{A \otimes_{R} q}\right)_{\frac{P}{A \otimes_{R} q}}\right) .
\end{aligned}
$$

Hence, by Lemma 2.4 as $q \cap R=n$ is maximal in $R$, we get

Since

$$
\lambda\left(\left(A \otimes_{R} B\right)_{P}\right)=\lambda(B)+\lambda\left(\left(\frac{A}{n A} \otimes_{k_{R}(n)} k_{B}(q)\right)_{P\left(\frac{A}{n A} \otimes_{k_{R}(n)} k_{B}(q)\right)}\right) .
$$

is flat, we have

$$
\frac{A}{n A} \longrightarrow\left(\frac{A}{n A} \otimes_{k_{R}(n)} k_{B}(q)\right)_{P\left(\frac{A_{p}}{n A_{p}} \otimes_{k_{R}(n)} k_{B}(q)\right)}
$$

$$
\lambda\left(\left(\frac{A}{n A} \otimes_{k_{R}(n)} k_{B}(q)\right)_{P\left(\frac{A}{n A} \otimes_{k_{R}(n)} k_{B}(q)\right)}\right)=\lambda\left(\frac{A}{n A}\right)+\lambda\left(\left(k_{A}(p) \otimes_{k_{R}(n)} k_{B}(q)\right)_{P\left(k_{A}(p) \otimes_{k_{R}(n)} k_{B}(q)\right)}\right) .
$$

It follows that

$$
\lambda\left(\left(A \otimes_{R} B\right)_{P}\right)=\lambda\left(\frac{A}{n A}\right)+\lambda(B)+\lambda\left(\left(k_{A}(p) \otimes_{k_{R}(n)} k_{B}(q)\right)_{P\left(k_{A}(p) \otimes_{k_{R}(n)} k_{B}(q)\right)}\right)
$$

completing the proof.

Corollary 2.5. $\Lambda=\Lambda^{\prime}$.

Proof. If $\lambda \in \Lambda$, with the notation of Theorem 2.3, we have

$$
\lambda\left(\frac{A_{p}}{n A_{p}}\right)+\lambda\left(R_{n}\right)=\lambda\left(A_{p}\right) .
$$

Then $\lambda \in \Lambda^{\prime}$, by Theorem 2.3 .

Now, if $\lambda^{\prime} \in \Lambda^{\prime}$, and $(S, m, k) \longrightarrow(T, n, l)$ is a flat local homomorphism, then

$$
\lambda^{\prime}(S)+\lambda^{\prime}\left(\frac{T}{m T}\right)=\lambda^{\prime}(S)+\lambda^{\prime}\left(T \otimes_{S} k\right)=\lambda^{\prime}(T)+\lambda^{\prime}(k)+\lambda^{\prime}\left(l \otimes_{k} k\right)=\lambda^{\prime}(T)
$$

since $\lambda^{\prime}$ vanishes on a field, so $\lambda^{\prime} \in \Lambda$.

Our next result deals with the Krull dimension as well as the depth of local tensor products of algebras over an arbitrary ring $R$. The case of a base field was obtained in [BK1, Proposition 2.3].

Corollary 2.6. Let $A$ and $B$ be R-algebras. Let $P$ be a prime ideal of $A \otimes_{R} B$ with $n:=P \cap R$, $p:=P \cap A$ and $q:=P \cap B$. Assume that $A_{p}$ is flat over $R_{n}$. Then

$$
\begin{gathered}
\text { (1) } \operatorname{dim}\left(\left(A \otimes_{R} B\right)_{P}\right)=\operatorname{dim}\left(A_{p}\right)+\operatorname{dim}\left(B_{q}\right)-\operatorname{dim}\left(R_{n}\right)+ \\
\operatorname{dim}\left(\left(k_{A}(p) \otimes_{k_{R}(n)} k_{B}(q)\right)_{P\left(k_{A}(p) \otimes_{k_{R}(n)} k_{B}(q)\right)}\right) . \\
\text { (2) } \operatorname{depth}\left(\left(A \otimes_{R} B\right)_{P}\right)=\operatorname{depth}\left(A_{p}\right)+\operatorname{depth}\left(B_{q}\right)-\operatorname{depth}\left(R_{n}\right)+ \\
\operatorname{dim}\left(\left(k_{A}(p) \otimes_{k_{R}(n)} k_{B}(q)\right)_{P\left(k_{A}(p) \otimes_{k_{R}(n)} k_{B}(q)\right)}\right) .
\end{gathered}
$$

Proof. By Proposition 2.2, $\operatorname{dim} \in \Lambda$ and depth $\in \Lambda$. Then the result follows from Corollary 2.5 since the Krull dimension and the depth are finite invariants when applied to Noetherian local rings, in other terms, dim, depth: $\mathcal{R} \longrightarrow \mathbb{N}$. 
Remark 2.7. The Krull dimension of $k_{A}(p) \otimes_{k_{R}(n)} k_{B}(q)$ is the minimum of the transcendence degrees of the field extensions $k_{A}(p) \mid k_{R}(n)$ and $k_{B}(q) \mid k_{R}(n)$. This was proved in [EGA IV, Quatriéme Partie, Err ${ }_{\mathrm{VI}}$.19, page 349]. See also [Sh2] and [Mu].

Recall that the "codepth" of a Noetherian local ring $A$ denoted by codepth $(A):=$ $\operatorname{dim}(A)-\operatorname{depth}(A)$ is an invariant introduced by Grothendieck in [EGA IV], Première Partie, $0_{\text {IV }}$.16.4.9] to measure the Cohen-Macaulayness defect of $A$. Next, we determine the codepth of local tensor products of algebras over a ring.

Corollary 2.8. Let $A$ and $B$ be R-algebras. Let $P$ be a prime ideal of $A \otimes_{R} B$ with $n:=P \cap R$, $p:=P \cap A$ and $q:=P \cap B$. Assume that $A_{p}$ is flat over $R_{n}$. Then

$$
\begin{aligned}
\operatorname{codepth}\left(\left(A \otimes_{R} B\right)_{P}\right) & =\operatorname{codepth}\left(A_{p}\right)+\operatorname{codepth}\left(B_{q}\right)-\operatorname{codepth}\left(R_{n}\right) \\
& =\operatorname{codepth}\left(\frac{A_{p}}{n A_{p}}\right)+\operatorname{codepth}\left(B_{q}\right) .
\end{aligned}
$$

Proof. The first equality follows from Corollary 2.6 and the second from Proposition 2.2.

We end this section by discussing the Cohen-Macaulayness of tensor products of algebras over a ring $R$. We begin with the local case and characterize when the localization of $A \otimes_{R} B$ is Cohen-Macaulay.

Corollary 2.9. Let $A$ and $B$ be R-algebras. Let $P$ be a prime ideal of $A \otimes_{R} B$ with $n:=P \cap R$, $p:=P \cap A$ and $q:=P \cap B$. Assume that $A_{p}$ is flat over $R_{n}$. Then the following assertions are equivalent:

(1) $\left(A \otimes_{R} B\right)_{P}$ is a Cohen-Macaulay ring;

(2) $\frac{A_{p}}{n A_{p}}$ and $B_{q}$ are Cohen-Macaulay rings;

(3) $B_{q}$ is a Cohen-Macaulay ring and $\operatorname{codepth}\left(A_{p}\right)=\operatorname{codepth}\left(R_{n}\right)$.

Proof. It is direct by the preceding corollary.

Corollary 2.10. Let $A$ and $B$ be R-algebras. Let $P$ be a prime ideal of $A \otimes_{R} B$ with $n:=P \cap R$, $p:=P \cap A$ and $q:=P \cap B$. Assume that $A_{p}$ and $B_{q}$ are flat over $R_{n}$. Then the following assertions are equivalent:

(1) $\left(A \otimes_{R} B\right)_{P}$ is a Cohen-Macaulay ring;

(2) $A_{p}$ and $B_{q}$ are Cohen-Macaulay rings.

Proof. (2) $\Rightarrow$ (1) It is direct by Corollary 2.9 as $R_{n} \longrightarrow A_{p}$ is a flat homomorphism. $(1) \Rightarrow(2)$ By the above corollary $B_{q}$ is Cohen-Macaulay, and then so is $A_{p}$ by symmetry.

Corollary 2.11. Let $A$ and $B$ be R-algebras. Let $P$ be a prime ideal of $A \otimes_{R} B$ with $n:=P \cap R$, $p:=P \cap A$ and $q:=P \cap B$. Assume that $A_{p}$ is flat over $R_{n}$ and $R_{n}$ is a Cohen-Macaulay ring. Then the following assertions are equivalent:

(1) $\left(A \otimes_{R} B\right)_{P}$ is a Cohen-Macaulay ring;

(2) $A_{p}$ and $B_{q}$ are Cohen-Macaulay rings.

Next, we deal with the global case of Cohen-Macaulayness of tensor products. The following corollaries generalize [BK1, Theorem 2.1] which characterizes the Cohen-Macaulayness of $A \otimes_{k} B$ in terms of the Cohen-Macaulayness of $A$ and $B$ in the setting of a field $k$. 
Corollary 2.12. Let $A$ and $B$ be R-algebras. Assume that $A$ is flat over $R$. Then the following assertions are equivalent:

(1) $A \otimes_{R} B$ is a Cohen-Macaulay ring;

(2) $\frac{A_{p}}{n A_{p}}$ and $B_{q}$ are Cohen-Macaulay rings for any prime ideals $p$ of $A$ and $q$ of $B$ such that $p \cap R=q \cap R=n$.

Proof. (1) $\Rightarrow$ (2) Assume that (1) holds. Let $p \in \operatorname{Spec}(A)$ and $q \in \operatorname{Spec}(B)$ such that $p \cap R=q \cap R=: n$. Then by Proposition 2.1 there exists a prime ideal $P$ of $A \otimes_{R} B$ such that $P \cap A=p$ and $P \cap B=q$. By (1), $A \otimes_{R} B$ is Cohen-Macaulay, thus $\left(A \otimes_{R} B\right)_{P}$ is Cohen-Macaulay. As a consequence, by Corollary 2.9. $B_{q}$ and $\frac{A_{p}}{n A_{p}}$ are CohenMacaulay.

(2) $\Rightarrow(1)$ Assume that (2) holds. Let $P$ be a prime ideal of $A \otimes_{R} B$ and let $n:=P \cap R$, $p:=P \cap A, q:=P \cap B$. As $p \cap R=q \cap R=P \cap R=n$, we get by (2) that $\frac{A_{p}}{n A_{p}}$ and $B_{q}$ are Cohen-Macaulay rings. Hence, by Corollary 2.9. $\left(A \otimes_{R} B\right)_{P}$ is Cohen-Macaulay. It follows that $A \otimes_{R} B$ is Cohen-Macaulay completing the proof.

Corollary 2.13. Let $A$ and $B$ be R-algebras. Assume that $A$ and $B$ are flat over $R$. Then the following assertions are equivalent:

(1) $A \otimes_{R} B$ is a Cohen-Macaulay ring;

(2) $A_{p}$ and $B_{q}$ are Cohen-Macaulay rings for any prime ideals $p$ of $A$ and $q$ of $B$ such that $p \cap R=q \cap R$.

Proof. It is similar to that of Corollary 2.12 using Corollary 2.10

Corollary 2.14. Let $R$ be a Cohen-Macaulay ring. Let $A$ and $B$ be R-algebras. Assume that $A$ is flat over $R$. Then the following assertions are equivalent:

(1) $A \otimes_{R} B$ is a Cohen-Macaulay ring;

(2) $A_{p}$ and $B_{q}$ are Cohen-Macaulay rings for any prime ideals $p$ of $A$ and $q$ of $B$ such that $p \cap R=q \cap R$.

Proof. Again, the proof is similar to that of Corollary 2.12, but using Corollary 2.11

\section{Gorenstein Rings}

The aim of this section is to determine the injective dimension of local tensor products of algebras over an arbitrary ring $R$. This permits to generalize [TY, Theorem 6] on Gorensteiness of tensor products of algebras over a field.

We begin by announcing the main theorem of this section. It gives the injective dimension of local tensor products of algebras over a ring $R$.

Theorem 3.1. Let $A$ and $B$ be $R$-algebras. Let $P$ be a prime ideal of $A \otimes_{R} B$ with $n:=P \cap R$, $p:=P \cap A$ and $q:=P \cap B$. Assume that $A_{p}$ is flat over $R_{n}$. Then

$\operatorname{id}_{\left(A \otimes_{R} B\right)_{P}}\left(\left(A \otimes_{R} B\right)_{P}\right)=\operatorname{id}_{\frac{A p}{n A_{p}}}\left(\frac{A_{p}}{n A_{p}}\right)+\operatorname{id}_{B_{q}}\left(B_{q}\right)+\operatorname{dim}\left(\left(k_{A}(p) \otimes_{k_{R}(n)} k_{B}(q)\right)_{P\left(k_{A}(p) \otimes_{k_{R}(n)} k_{B}(q)\right)}\right)$.

Proof. The proof is straightforward from Theorem 2.3 since, by Proposition 2.2 id $\in \Lambda$, and using Bass's formula [B, Lemma 3.3] we have that

$$
\operatorname{id}_{D}(D)=\operatorname{depth}(D)=\operatorname{dim}(D),
$$


as $D:=\left(k_{A}(p) \otimes_{k} k_{B}(q)\right)_{P\left(k_{A}(p) \otimes_{k} k_{B}(q)\right)}$ is a Gorenstein local ring by [TY, Theorem 6].

The formula for the injective dimension of local tensor products of algebras over a field $k$ is simpler as recorded next.

Corollary 3.2. Let $k$ be a field. Let $A$ and $B$ be k-algebras. Let $P$ be a prime ideal of $A \otimes_{k} B$ with $p:=P \cap A$ and $q:=P \cap B$. Then

$$
\operatorname{id}_{\left(A \otimes_{k} B\right)_{P}}\left(\left(A \otimes_{k} B\right)_{P}\right)=\operatorname{id}_{A_{p}}\left(A_{p}\right)+\operatorname{id}_{B_{q}}\left(B_{q}\right)+\operatorname{dim}\left(\left(k_{A}(p) \otimes_{k} k_{B}(q)\right)_{P\left(k_{A}(p) \otimes_{k} k_{B}(q)\right)}\right) .
$$

The remaining of this section is devoted to discuss the Gorensteiness of tensor products of algebras over a ring $R$ in the local case as well as in the global one. In particular, we generalize [TY, Theorem 6] which proves that a tensor product $A \otimes_{k} B$ of algebras $A$ and $B$ over a field $k$ is Gorenstein if and only if $A$ and $B$ are so. The following corollaries are direct consequences of Theorem 3.1 so that the proofs are omitted. It suffices to recall that $\left(k_{A}(p) \otimes_{k_{R}(n)} k_{B}(q)\right)_{P\left(k_{A}(p) \otimes_{k_{R}(n)} k_{B}(q)\right)}$ is a Gorenstein local ring.

Corollary 3.3. Let $A$ and $B$ be R-algebras. Let $P \in \operatorname{Spec}\left(A \otimes_{R} B\right)$ and let $n:=P \cap R$, $p:=P \cap A$ and $q:=P \cap B$. Assume that $R_{n} \longrightarrow A_{p}$ is a flat ring homomorphism. Then the following assertions are equivalent:

(1) $\left(A \otimes_{R} B\right)_{P}$ is a Gorenstein ring;

(2) $\frac{A_{p}}{n A_{p}}$ and $B_{q}$ are Gorenstein rings.

Corollary 3.4. Let $A$ and $B$ be R-algebras. Let $P \in \operatorname{Spec}\left(A \otimes_{R} B\right)$ and let $n:=P \cap R$, $p:=P \cap A$ and $q:=P \cap B$. Assume that $R_{n} \longrightarrow A_{p}$ is a flat ring homomorphism and that $R_{n}$ is a Gorenstein ring. Then the following assertions are equivalent:

(1) $\left(A \otimes_{R} B\right)_{P}$ is a Gorenstein ring;

(2) $A_{p}$ and $B_{q}$ are Gorenstein rings.

Corollary 3.5. Let $A$ and $B$ be R-algebras. Assume that $R \longrightarrow A$ is a flat ring homomorphism. Then the following assertions are equivalent:

(1) $A \otimes_{R} B$ is a Gorenstein ring;

(2) $\frac{A_{p}}{n A_{p}}$ and $B_{q}$ are Gorenstein rings for any prime ideals $p$ of $A$ and $q$ of $B$ such that $p \cap R=q \cap R$.

Corollary 3.6. Let $R$ be a Gorenstein ring. Let $A$ and $B$ be R-algebras. Assume that $A$ and $B$ are flat over $R$. Then the following assertions are equivalent:

(1) $A \otimes_{R} B$ is a Gorenstein ring;

(2) $A_{p}$ and $B_{q}$ are Gorenstein rings for any prime ideals $p$ of $A$ and $q$ of $B$ such that $p \cap R=q \cap R$.

\section{TYPE OF LOCAL RINGS}

Let $(R, m, k)$ be a local ring, where $m$ is the maximal ideal of $R$ and $k$ its residue field. Recall that the type of an $R$-module $M$, denoted by $r_{R}(M)$, is an invariant which refines the information given by the depth of $M$, namely $r_{R}(M):=\operatorname{dim}_{k}\left(\operatorname{Ext}_{R}^{t}(k, M)\right)$ where $t=\operatorname{depth}_{R}(M)$. In this section, we express the type of the tensor product of algebras over a ring in terms of the type of its components. 
We begin by recording the following theorem which examines the behavior of the type under flat ring homomorphisms.

Theorem 4.1. Let $(R, m, k) \longrightarrow(S, n, l)$ be a flat ring homomorphism of local Noetherian rings. Then

$$
r_{S}(S)=r_{R}(R) \cdot r_{\frac{S}{m S}}\left(\frac{S}{m S}\right) .
$$

Proof. See [FT, Theorem].

The following result is a direct consequence of this theorem.

Corollary 4.2. The composed function $\ln$ or of the the Neperian logarithm $\ln$ and the type $r$ is an element of $\Lambda$.

Recall that, by $[\overline{\mathrm{BH}}$, Theorem 3.2.10], a local ring $A$ is Gorenstein if and only if $A$ is a Cohen-Macaulay ring of type 11. From this perspective, the type of local rings might be regarded as an invariant which measures the defect of Gorensteiness of local rings. The following result examines the behavior of the property of being "of type 1" under flat ring homomorphisms.

Corollary 4.3. Let $(R, m, k) \longrightarrow(S, n, l)$ be a flat ring homomorphism of local Noetherian rings. Then $S$ is of type 1 if and only if so are $R$ and $\frac{S}{m S}$.

Proof. The proof is straightforward from Theorem 4.1

Next, we announce the main theorem of this section. It computes the type of a local tensor product of algebras over a ring $R$ in terms of the types of their components. Given a ring $A$ and when no confusion is likely, we denote by $r(A)$ the type $r_{A}(A)$ of $A$ over itself.

Theorem 4.4. Let $A$ and $B$ be R-algebras. Let $P$ be a prime ideal of $A \otimes_{R} B$ and let $n:=P \cap R, p:=P \cap A$ and $q:=P \cap B$. Assume that $A_{p}$ is flat over $R_{n}$. Then

$$
r\left(\left(A \otimes_{R} B\right)_{P}\right)=r\left(\frac{A_{p}}{n A_{p}}\right) \cdot r\left(B_{q}\right)=\frac{r\left(A_{p}\right) \cdot r\left(B_{q}\right)}{r\left(R_{n}\right)} .
$$

Proof. The proof follows from Theorem 4.1, Theorem 2.3 and Corollary 4.2, noting that $\left(k_{A}(p) \otimes_{k_{R}(n)} k_{B}(q)\right)_{P k_{A}(p) \otimes_{k_{R}(n)} k_{B}(q)}$ is a Gorenstein local ring and thus of type 1 .

Now, we record when a local tensor product of algebras over a ring behaves nicely with respect to Gorensteiness, namely when it is of type 1.

Corollary 4.5. Let $A$ and $B$ be R-algebras. Let $P$ be a prime ideal of $A \otimes_{R} B$ and let $n:=P \cap R, p:=P \cap A$ and $q:=P \cap B$. Assume that $A_{p}$ is flat over $R_{n}$. Then $\left(A \otimes_{R} B\right)_{P}$ is of type 1 if and only if $\frac{A_{p}}{n A_{p}}$ and $B_{q}$ are of type 1 .

Proof. It is clear from Theorem 4.4

Our last results of this section determine the type of local tensor products of algebras over a field $k$ and examine when these constructions are of type 1.

\footnotetext{
${ }^{1}$ Note that if the type is defined as $r_{R}(R):=\operatorname{dim}_{k}\left(\operatorname{Ext}_{R}^{t}(k, R)\right)$ where $t=\operatorname{dim} R$ instead of $t=\operatorname{depth}_{R}(R)$, then a local ring is Gorenstein if and only if it is of type $1[\mathbb{R}]$.
} 
Corollary 4.6. Let $k$ be a field and let $A$ and $B$ be $k$-algebras. Let $P$ be a prime ideal of $A \otimes_{k} B$. Let $p:=P \cap A$ and $q:=P \cap B$. Then

$$
r\left(\left(A \otimes_{k} B\right)_{P}\right)=r\left(A_{p}\right) \cdot r\left(B_{q}\right) .
$$

Corollary 4.7. Let $k$ be $a$ field and let $A$ and $B$ be $k$-algebras. Let $P$ be a prime ideal of $A \otimes_{k} B$. Let $p:=P \cap A$ and $q:=P \cap B$. Then $\left(A \otimes_{k} B\right)_{P}$ is of type 1 if and only if $A_{p}$ and $B_{q}$ are of type 1 .

\section{Introduction to André-Quillen homology}

This section represents a short introduction to André-Quillen homology. If $A \longrightarrow B$ is a ring homomorphism and $M$ is a $B$-module, then $H_{n}(A, B, M)$ denotes the André-Quillen homology $B$-module for each $n \geq 0$ [An]. In the following, we list the basic properties of these homology modules used in the remaining sections.

Properties. (i) (Functoriality) They are natural in all three variables $A, B$ and $M$.

(ii) If $A \longrightarrow B$ is an isomorphism, then $H_{n}(A, B, M)=0$ for all $n \geq 0$. If $B \cong \frac{A}{I}$, then $H_{0}(A, B, M)=0$ and

$$
H_{1}(A, B, M) \cong \frac{I}{I^{2}} \otimes_{B} M .
$$

In particular, if $(A, m, k)$ is a Noetherian local ring, $\operatorname{dim}_{F} H_{1}(A, k, F)=\operatorname{embdim}(A)$ for any field extension $F \mid k$.

(iii) (Base change) Let $A \longrightarrow B, A \longrightarrow C$ be ring homomorphisms and $t$ an integer such that $\operatorname{Tor}_{n}^{A}(B, C)=0$ for all $0<n<t$. Then for any $B \otimes_{A} C$-module $M$, the canonical homomorphisms

$$
H_{n}(A, B, M) \longrightarrow H_{n}\left(C, B \otimes_{A} C, M\right)
$$

are isomorphisms for all $n<t$.

(iv) Let $A \longrightarrow B \longrightarrow C$ be ring homomorphisms and $M$ a flat $C$-module. Then

$$
H_{n}(A, B, M) \cong H_{n}(A, B, C) \otimes_{C} M
$$

for all $n$.

(v) (Jacobi-Zariski exact sequence) Let $A \longrightarrow B \longrightarrow C$ be ring homomorphisms and $M a$ $C$-module. Then there exist a natural exact sequence

$$
\begin{aligned}
\cdots & \longrightarrow H_{n}(A, B, M) \longrightarrow H_{n}(A, C, M) \longrightarrow H_{n}(B, C, M) \longrightarrow \\
& \longrightarrow H_{n-1}(A, B, M) \longrightarrow \cdots \\
\cdots & \longrightarrow H_{0}(A, B, M) \longrightarrow H_{0}(A, C, M) \longrightarrow H_{0}(B, C, M) \longrightarrow 0
\end{aligned}
$$

(vi) (Tensor product) Let $B$ and $C$ be A-algebras and $t$ an integer such that $\operatorname{Tor}_{n}^{A}(B, C)=0$ for all $0<n<t$. Then for any $B \otimes_{A} C$-algebra $D$ and any $D$-module $M$ we have a natural exact sequence

$$
\begin{aligned}
\longrightarrow H_{t-1}(A, D, M) \longrightarrow H_{t-1}(B, D, M) \oplus H_{t-1}(C, D, M) & \longrightarrow H_{t-1}\left(B \otimes_{A} C, D, M\right) \longrightarrow \\
\cdots & \longrightarrow H_{0}\left(B \otimes_{A} C, D, M\right) \longrightarrow \cdots \\
& \longrightarrow 0
\end{aligned}
$$

(vii) (Field extensions) If $L \mid K$ is a field extension and $M$ an $L$-module, then $H_{n}(K, L, M)=0$ for all $n \geq 2$. Moreover, $L \mid K$ is separable if and only if $H_{1}(K, L, M)=0$ for some (equivalently any) $L$-module $M \neq 0$. Therefore if $A \longrightarrow K \longrightarrow L$ are ring homomorphisms with $K$ and $L$ fields, then by $(v) H_{n}(A, K, M) \longrightarrow H_{n}(A, L, M)$ is an isomorphism for all $n \geq 2$ and 
injective for $n=1$. If $L \mid K$ is separable, it is also an isomorphism for $n=1$.

(viii) (Localization) Let $f: A \longrightarrow B$ be a ring homomorphism and let $S \subset A, T \subset B$ be multiplicatively closed subsets such that $f(S) \subset T$. Let $M$ be a B-module, then

$$
\begin{aligned}
T^{-1} H_{n}(A, B, M) & \cong H_{n}\left(A, B, T^{-1} M\right) \cong H_{n}\left(A, T^{-1} B, T^{-1} M\right) \\
& \cong H_{n}\left(S^{-1} A, T^{-1} B, T^{-1} M\right)
\end{aligned}
$$

for all $n \geq 0$.

(ix) (Finiteness) If $B$ an A-algebra of finite type and $M a$ B-module of finite type, then $H_{n}(A, B, M)$ is a B-module of finite type for all $n \geq 0$.

$(x)$ (Vanishing) A Noetherian local ring $(A, m, k)$ is regular if and only if $H_{2}(A, k, k)=0$ if and only if $H_{n}(A, k, k)=0$ for all $n \geq 2$. If $A$ is a ring and $(B, n, l)$ a local $A$-algebra, then $B$ is formally smooth for the $n$-adic topology [EGA IV], Première Partie, $0_{\text {IV.19.3.1] if and }}$ only if $H_{1}(A, B, l)=0$.

(xi) (Flat extensions) If $(A, m, k) \longrightarrow(B, n, l)$ is a local flat homomorphism of local rings, then the homomorphism $\mathrm{H}_{2}(A, k, l) \longrightarrow \mathrm{H}_{2}(B, l, l)$ is injective.

(xii) (Completions) Let $(A, m, k)$ and $(B, n, l)$ be two local rings with their completions $\hat{A}$ and $\hat{B}$. Then

$$
H_{n}(A, k, k) \cong H_{n}(\hat{A}, k, k) \quad \text { and } \quad H_{n}(A, B, l) \cong H_{n}(\hat{A}, \hat{B}, l)
$$

for all $n$.

Proof. (i) See [An, 3.15].

(ii) See $[\mathrm{An}, 4.43,4.60,6.1]$.

(iii) See [An, 9.31].

(iv) See [An, 3.20].

(v) See [An, 5.1].

(vi) Same proof than in [An, 5.21], but using [An, 9.31] instead of [An, 4.54].

(vii) See [An. 7.4, 7.13].

(viii) The first isomorphism is [An, 4.59] and the last two follow from [An, 5.27].

(ix) See [An, 4.55].

(x) [An, 6.26] gives the first claim. The second follows from [An, 16.17, 3.20, 3.21].

(xi) See [Av, Remarks (1.4)].

(xii) The first isomorphism is [An, 10.18] and the second one is an immediate consequence of the first (a reference for this isomorphism is [FR, Lemma 1]).

\section{Complete intersection}

The aim of this section is to evaluate the complete intersection defect of the tensor product of two algebras over a ring.

Let $(S, m, l)$ be a local ring. The complete intersection defect of $S[\overline{\mathrm{KK}}]$ is defined as

$$
\mathrm{d}(S):=\varepsilon_{2}(S)-\operatorname{embdim}(S)+\operatorname{dim}(S),
$$

where $\varepsilon_{2}(S)$ denotes $\operatorname{dim}_{l} H_{2}(S, l, l)$, which is finite by Property (ix). It is remarkable that, by [An, 15.12] (see also [MR, 2.5.1]), $\varepsilon_{2}(S)$ coincides with the dimension of the first Koszul homology module, denoted by $\operatorname{dim} H_{1}(m)$, associated to a minimal set of generators of the ideal $m$. Also, notice that $\mathrm{d}(S) \geq 0$, and $\mathrm{d}(S)=0$ if and only if $S$ is complete intersection [KK, p. 349] (or in terms of André-Quillen homology see [MR, 4.3.4, 4.3.5]).

Recall that, in [M, Theorem 2], it is proved the following theorem on complete intersection of tensor products: Let $A$ and $B$ two $R$-algebras such that for each maximal 
ideal $P$ of $A \otimes_{R} B$, with $n=P \cap R, p=P \cap A$ and $q=P \cap B, A_{p}$ or $B_{q}$ is flat over $R_{n}$. If $A$ and $B$ are complete intersection then so is $A \otimes_{R} B$. Our next theorem generalizes this result by evaluating the complete intersection defect of such constructions.

Theorem 6.1. Let $A$ and $B$ be $R$-algebras. Let $P \in \operatorname{Spec}\left(A \otimes_{R} B\right)$ with $p:=P \cap A, q:=P \cap B$ and $n:=P \cap R$. Assume that $A_{p}$ is flat over $R_{n}$. Then

$$
d\left(\left(A \otimes_{R} B\right)_{P}\right)=d\left(A_{p}\right)+d\left(B_{q}\right)-d\left(R_{n}\right) .
$$

Proof. It is immediate from Proposition 2.2 and Corollary 2.5 as

$\left(k_{A}(p) \otimes_{k_{R}(n)} k_{B}(q)\right)_{P\left(k_{A}(p) \otimes_{k_{R}(n)} k_{B}(q)\right)}$ is complete intersection.

Next, we isolate the case of tensor products of algebras over a field.

Corollary 6.2. Let $k$ be a field and let $A$ and $B$ be k-algebras. Let $P \in \operatorname{Spec}\left(A \otimes_{k} B\right)$ with $p:=P \cap A$ and $q:=P \cap B$. Then

$$
\mathrm{d}\left(\left(A \otimes_{k} B\right)_{P}\right)=\mathrm{d}\left(A_{p}\right)+\mathrm{d}\left(B_{q}\right) .
$$

The next corollaries characterizes when the tensor product of algebras over a ring is complete intersection.

Corollary 6.3. Let $A$ and $B$ be R-algebras. Let $P \in \operatorname{Spec}\left(A \otimes_{R} B\right)$ with $p:=P \cap A$, $q:=P \cap B$ and $n:=P \cap R$. Assume that $A_{p}$ is flat over $R_{n}$. Then the following assertions are equivalent:

(1) $\left(A \otimes_{R} B\right)_{P}$ is complete intersection;

(2) $\frac{A_{p}}{n A_{p}}$ and $B_{q}$ are complete intersections;

(3) $B_{q}$ is complete intersection and $\mathrm{d}\left(A_{p}\right)=\mathrm{d}\left(R_{n}\right)$.

Proof. It suffices to note, by Theorem 6.1, that $\mathrm{d}\left(\left(A \otimes_{R} B\right)_{P}\right)=\mathrm{d}\left(\frac{A_{p}}{n A_{p}}\right)+\mathrm{d}\left(B_{q}\right)$.

Corollary 6.4. Let $A$ and $B$ be R-algebras. Let $P \in \operatorname{Spec}\left(A \otimes_{R} B\right)$ with $p:=P \cap A, q:=P \cap B$ and $n:=P \cap R$. Assume that $A_{p}$ and $B_{q}$ are flat over $R_{n}$. Then the following assertions are equivalent:

(1) $\left(A \otimes_{R} B\right)_{P}$ is complete intersection;

(2) $A_{p}$ and $B_{q}$ are complete intersections.

Proof. It is direct as if either $A_{p}$ or $B_{q}$ is complete intersection, then so is $R_{n}$.

Note that one may characterize when the tensor product $A \otimes_{R} B$ is complete intersection by using Proposition 2.1 as done in Section 2 for Cohen-Macaulayness.

Let $\left(R, n, k_{R}(n)\right)$ be a Noetherian local ring and $\hat{R}$ its completion. It is well known (see $[\mathrm{K}]$ ) that for any representation of $\hat{R}$ as $\hat{R}=\frac{S}{I}$, where $S$ is a regular local ring and $I$ an ideal of $R$, we have

$$
\mathrm{d}(R)=\mu(I)-\operatorname{dim}(S)+\operatorname{dim}(R) .
$$

Here, $\mu(I)=\operatorname{dim} H_{1}\left(S, \hat{R}, k_{R}(n)\right)$ stands for the minimum number of generators of $I$ (Property (ii)). $R$ is said to be an almost complete intersection ring if $\mathrm{d}(R)=1$.

The following two corollaries characterize when the tensor product of two $R$ algebras is almost complete intersection. 
Corollary 6.5. Let $A$ and $B$ be R-algebras. Let $P \in \operatorname{Spec}\left(A \otimes_{R} B\right)$ with $p:=P \cap A$, $q:=P \cap B$ and $n:=P \cap R$. Assume that $A_{p}$ is flat over $R_{n}$. Then $\left(A \otimes_{R} B\right)_{P}$ is almost complete intersection if and only if one of the following assertions holds:

(1) $\frac{A_{p}}{n A_{p}}$ is complete intersection and $B_{q}$ is almost complete intersection.

(2) $\frac{A_{p}}{n A_{p}}$ is almost complete intersection and $B_{q}$ is complete intersection.

Proof. It is clear as by Theorem 6.1, $\mathrm{d}\left(\left(A \otimes_{R} B\right)_{P}\right)=\mathrm{d}\left(\frac{A_{p}}{n A_{p}}\right)+d\left(B_{q}\right)$.

Note that under the conditions of Corollary 6.5 if $R_{n}, A_{p}$ and $B_{q}$ are almost complete intersection, then so is $\left(A \otimes_{R} B\right)_{P}$.

Corollary 6.6. Let $A$ and $B$ be R-algebras. Let $P \in \operatorname{Spec}\left(A \otimes_{R} B\right)$ with $p:=P \cap A, q:=P \cap B$ and $n:=P \cap R$. Assume that $A_{p}$ and $B_{q}$ are flat over $R_{n}$. Then $\left(A \otimes_{R} B\right)_{P}$ is almost complete intersection if and only if one of the following assertions holds:

(1) $R_{n}, A_{p}$ and $B_{q}$ are almost complete intersection rings.

(2) $R_{n}$ and $A_{p}$ are complete intersection and $B_{q}$ is almost complete intersection.

(3) $R_{n}$ and $B_{q}$ are complete intersection and $A_{p}$ is almost complete intersection.

Proof. First, as by Theorem 6.1, $\mathrm{d}\left(\left(A \otimes_{R} B\right)_{P}\right)=\mathrm{d}\left(\frac{A_{p}}{n A_{p}}\right)+\mathrm{d}\left(B_{q}\right)=\mathrm{d}\left(A_{p}\right)+\mathrm{d}\left(\frac{B_{q}}{n B_{q}}\right)$, note that if $\left(A \otimes_{R} B\right)_{P}$ is almost complete intersection, then $\mathrm{d}\left(R_{n}\right) \leq 1, \mathrm{~d}\left(A_{p}\right) \leq 1$ and $\mathrm{d}\left(B_{q}\right) \leq 1$. If $R_{n}$ is complete intersection, then, by Corollary 6.5. $\left(A \otimes_{R} B\right)_{P}$ is almost complete intersection if and only if either $A_{p}$ is almost complete intersection and $B_{q}$ is complete intersection or $A_{p}$ is complete intersection and $B_{q}$ is almost complete intersection, as desired. Now, assume that $R_{n}$ is almost complete intersection. As $\mathrm{d}\left(A_{p}\right)=\mathrm{d}\left(R_{n}\right)+\mathrm{d}\left(\frac{A_{p}}{n A_{p}}\right) \geq 1$, if $\mathrm{d}\left(A_{p}\right) \leq 1$, then $\mathrm{d}\left(A_{p}\right)=1$, that is, $A_{p}$ is almost complete intersection. Similarly, if $\mathrm{d}\left(B_{q}\right) \leq 1$, then $B_{q}$ is almost complete intersection. It follows that $\left(A \otimes_{R} B\right)_{P}$ is almost complete intersection if and only if $A_{p}$ and $B_{q}$ are so. This completes the proof of the corollary.

\section{Regular Rings}

We are mainly concerned in this section with measuring the defect of regularity of the tensor product of algebras over a ring.

For a local ring $S$, we denote by $\operatorname{codim} S:=\operatorname{emb} \operatorname{dim} S-\operatorname{dim} S$ the codimension of $S$, so that $S$ is regular if and only if $\operatorname{codim} S=0$. Thereby, from the very definition of complete intersection defect of $S$, note that

$$
\varepsilon_{2}(S)=\mathrm{d}(S)+\operatorname{codim}(S)
$$

where $\varepsilon_{2}(S)=\operatorname{dim}_{l} H_{2}(S, l, l)$. Therefore $\varepsilon_{2}(S)$, as well as $\operatorname{codim}(S)$, is a measure of the defect of regularity of $S$ (it also follows from Property $(\mathrm{x})$ ).

Lemma 7.1. Let $A$ and $B$ be two R-algebras. Let $P$ be a prime ideal of $A \otimes_{R} B$ with $n:=P \cap R, p:=P \cap A$ and $q:=P \cap B$. Assume that $A_{p}$ is a flat $R_{n}$-module. Then, the following statements are equivalent:

(1) $\varepsilon_{2}\left(\left(A \otimes_{R} B\right)_{P}\right)=\varepsilon_{2}\left(A_{p}\right)+\varepsilon_{2}\left(B_{q}\right)-\varepsilon_{2}\left(R_{n}\right)$;

(2) $\operatorname{codim}\left(\left(A \otimes_{R} B\right)_{P}\right)=\operatorname{codim}\left(A_{p}\right)+\operatorname{codim}\left(B_{q}\right)-\operatorname{codim}\left(R_{n}\right)$. 
Proof. It suffices to note that, by Theorem 6.1.

$$
\mathrm{d}\left(\left(A \otimes_{R} B\right)_{P}\right)=\mathrm{d}\left(A_{p}\right)+\mathrm{d}\left(B_{q}\right)-\mathrm{d}\left(R_{n}\right)
$$

and thus

$$
\begin{aligned}
\varepsilon_{2}\left(\left(A \otimes_{R} B\right)_{P}\right)-\varepsilon_{2}\left(A_{p}\right)-\varepsilon_{2}\left(B_{q}\right)+\varepsilon_{2}\left(R_{n}\right)= & \operatorname{codim}\left(\left(A \otimes_{R} B\right)_{P}\right)-\operatorname{codim}\left(A_{p}\right) \\
& -\operatorname{codim}\left(B_{q}\right)+\operatorname{codim}\left(R_{n}\right) .
\end{aligned}
$$

Recently, in [M, Theorem 1], it is proved the following theorem on regularity of tensor products: Let $R$ be a Noetherian ring and let $A$ and $B$ be two Noetherian $R$-algebras such that $A \otimes_{R} B$ is a Noetherian ring. Assume that for each maximal ideal $P$ of $A \otimes_{R} B$ with $n=P \cap R, q=P \cap A$ and $q=P \cap B$ at least one of the three local $R_{n}$-algebras $A_{p}, B_{q}$ or $k_{A \otimes_{R} B}(P)$ is formally smooth for the topology of its maximal ideal. If $A$ and $B$ are regular rings, then so is $A \otimes_{R} B$. Our next results generalize this theorem by measuring the defect of regularity of these tensor products under the formal smoothness of one the components over the base ring.

We begin by dealing with the codimension of rings issued from formally smooth homomorphisms.

Theorem 7.2. Let $A$ and $B$ be two R-algebras. Let $P$ be a prime ideal of $A \otimes_{R} B$ with $n:=P \cap R, p:=P \cap A$ and $q:=P \cap B$. If $R_{n} \longrightarrow A_{p}$ is formally smooth, then

$$
\operatorname{codim}\left(\left(A \otimes_{R} B\right)_{P}\right)=\operatorname{codim}\left(A_{p}\right)+\operatorname{codim}\left(B_{q}\right)-\operatorname{codim}\left(R_{n}\right) .
$$

Proof. Assume that $R_{n} \longrightarrow A_{p}$ is formally smooth. In light of Lemma7.1 it suffices to prove that $\varepsilon_{2}\left(\left(A \otimes_{R} B\right)_{P}\right)=\varepsilon_{2}\left(A_{p}\right)+\varepsilon_{2}\left(B_{q}\right)-\varepsilon_{2}\left(R_{n}\right)$. Observe that, by [EGA IV] Première Partie, $0_{\mathrm{IV}}$.19.7.1], $A_{p}$ is a flat $R_{n}$-module. Now, using Property (vi), consider the exact sequence (where $E$ is the residue field of $\left(A \otimes_{R} B\right)_{P}$ )

$$
\begin{aligned}
& H_{2}\left(R_{n}, E, E\right) \stackrel{\alpha_{2}}{\longrightarrow} H_{2}\left(A_{p}, E, E\right) \oplus H_{2}\left(B_{q}, E, E\right) \longrightarrow H_{2}\left(A_{p} \otimes_{R_{n}} B_{q}, E, E\right) \longrightarrow \\
\longrightarrow & H_{1}\left(R_{n}, E, E\right) \stackrel{\alpha_{1}}{\longrightarrow} H_{1}\left(A_{p}, E, E\right) \oplus H_{1}\left(B_{q}, E, E\right) .
\end{aligned}
$$

Let us show that $\alpha_{1}$ and $\alpha_{2}$ are injective. Note that $\alpha_{2}$ is injective by Property (xi). Also, Property (v) yields the following exact sequence

$$
H_{1}\left(R_{n}, A_{p}, E\right)=0 \stackrel{\beta}{\longrightarrow} H_{1}\left(R_{n}, E, E\right) \longrightarrow H_{1}\left(A_{p}, E, E\right)
$$

by Property (x) as $R_{n} \longrightarrow A_{p}$ is formally smooth. Therefore $\alpha_{1}$ is injective. Hence the following sequence is exact

$$
0 \longrightarrow H_{2}\left(R_{n}, E, E\right) \longrightarrow H_{2}\left(A_{p}, E, E\right) \oplus H_{2}\left(B_{q}, E, E\right) \longrightarrow H_{2}\left(A_{p} \otimes_{R_{n}} B_{q}, E, E\right) \longrightarrow 0
$$

As $H_{2}\left(A_{p} \otimes_{R_{n}} B_{q}, E, E\right) \cong H_{2}\left(\left(A \otimes_{R} B\right)_{P}, E, E\right)$ by Property (viii), and $\operatorname{dim}_{E} H_{2}\left(A_{p}, E, E\right)=$ $\varepsilon_{2}\left(A_{p}\right)$ by Property (vii) (and similarly for $R_{n}$ and $B_{q}$ ), it follows that

$$
\varepsilon_{2}\left(\left(A \otimes_{R} B\right)_{P}\right)=\varepsilon_{2}\left(A_{p}\right)+\varepsilon_{2}\left(B_{q}\right)-\varepsilon_{2}\left(R_{n}\right)
$$

completing the proof of the theorem.

Corollary 7.3. Let $(A, m, k) \longrightarrow(B, n, l)$ be a formally smooth homomorphism of local rings. Then

$$
\begin{aligned}
& \text { (1) } \varepsilon_{2}(B)=\varepsilon_{2}(A)+\varepsilon_{2}\left(\frac{B}{m B}\right) . \\
& \text { (2) } \operatorname{codim}(B)=\operatorname{codim}(A)+\operatorname{codim}\left(\frac{B}{m B}\right) .
\end{aligned}
$$


Proof. Part (2) follows from Theorem 7.2 and Lemma 7.1, since $\operatorname{codim}\left(\frac{B}{m B}\right)=\operatorname{codim}\left(B \otimes_{A} k\right)=\operatorname{codim} B-\operatorname{codim} A+\operatorname{codim} k=\operatorname{codim} B-\operatorname{codim} A$ and (1) follows from (2) as above.

The following corollaries discuss the regularity property as well as the embedding dimension of the tensor products of algebras over a ring under formal smoothness hypothesis.

Corollary 7.4. Let $A$ and $B$ be two R-algebras. Let $P$ be a prime ideal of $A \otimes_{R} B$ with $n:=P \cap R, p:=P \cap A$ and $q:=P \cap B$. Assume that $R_{n} \longrightarrow A_{p}$ is formally smooth. Consider the following assertions:

(1) $A_{p}$ and $B_{q}$ are regular.

(2) $\left(A \otimes_{R} B\right)_{P}$ is regular;

(3) $\frac{A_{p}}{n A_{p}}$ and $B_{q}$ are regular;

(4) $B_{q}$ is regular and $\operatorname{codim}\left(A_{p}\right)=\operatorname{codim}\left(R_{n}\right)$.

Then $(1) \Rightarrow(2) \Leftrightarrow(3) \Leftrightarrow(4)$.

Proof. It is direct from Theorem 7.2 as by Corollary $7.3, \operatorname{codim}\left(A_{p}\right)=\operatorname{codim}\left(R_{n}\right)+$ $\operatorname{codim}\left(\frac{A_{p}}{n A_{p}}\right)$

Corollary 7.5. Let $A$ and $B$ be two R-algebras. Let $P$ be a prime ideal of $A \otimes_{R} B$ with $n:=P \cap R, p:=P \cap A$ and $q:=P \cap B$. If $R_{n} \longrightarrow A_{p}$ is formally smooth, then $\operatorname{embdim}\left(\left(A \otimes_{R} B\right)_{P}\right)=\operatorname{embdim}\left(A_{p}\right)+\operatorname{embdim}\left(B_{q}\right)-\operatorname{embdim}\left(R_{n}\right)$

$$
+\operatorname{dim}\left(\left(k_{A}(p) \otimes_{k_{R}(n)} k_{B}(q)\right)_{P\left(k_{A}(p) \otimes_{k_{R}(n)} k_{B}(q)\right)}\right) .
$$

Proof. It follows from Corollary 2.6 and Theorem 7.2

Next, we announce our second main theorem of this section.

Theorem 7.6. Let $A$ and $B$ be $R$-algebras. Let $P$ be a prime ideal of $A \otimes_{R} B$ with $p:=P \cap A$ and $q:=P \cap B$. Assume that $R_{n} \longrightarrow k_{A}(p)$ is formally smooth, that is, $R_{n}$ is a field and $k_{A}(p)$ is separable over $R_{n}$. Then

(1) $\operatorname{codim}\left(\left(A \otimes_{R} B\right)_{P}\right)=\operatorname{codim}\left(A_{p}\right)+\operatorname{codim}\left(B_{q}\right)$.

(2) $\operatorname{embdim}\left(\left(A \otimes_{R} B\right)_{P}\right)=\operatorname{embdim}\left(A_{p}\right)+\operatorname{embdim}\left(B_{q}\right)$

$$
+\operatorname{dim}\left(\left(k_{A}(p) \otimes_{R_{n}} k_{B}(q)\right)_{P\left(k_{A}(p) \otimes_{R_{n}} k_{B}(q)\right)}\right) .
$$

Proof. The proof of (1) is the same as that of Theorem 7.2, taking in mind that the homomorphism $\beta$ in that proof factorizes as

$$
H_{1}\left(R_{n}, A_{p}, E\right) \longrightarrow H_{1}\left(R_{n}, k_{A}(p), E\right) \longrightarrow H_{1}\left(R_{n}, E, E\right)
$$

and $H_{1}\left(R_{n}, k_{A}(p), E\right)=0$ by Property (vii). Then (2) follows from (1) and Corollary 2.6 .

Corollary 7.7. Let $A$ and $B$ be R-algebras. Let $P$ be a prime ideal of $A \otimes_{R} B$ with $p:=P \cap A$ and $q:=P \cap B$. Assume that $R_{n} \longrightarrow k_{A}(p)$ is formally smooth (i.e., $R_{n}$ is a field and $k_{A}(p)$ is separable over $\left.R_{n}\right)$. Then the following assertions are equivalent:

(1) $\left(A \otimes_{R} B\right)_{P}$ is regular;

(2) $A_{p}$ and $B_{q}$ are regular. 


\section{REFERENCES}

[An] André, M. Homologie des algébres commutatives. Grundlehren Math. Wiss., 206. SpringerVerlag, Berlin-New York, 1974.

[Av] Avramov, L.L. Homology of local flat extensions and complete intersection defects. Math. Ann., 228(1) (1977), 27-37.

[B] Bass, H. On the ubiquity of Gorenstein rings. Math. Z., 82 (1963), 8-28.

[BK1] Bouchiba, S.; Kabbaj, S. Tensor products of Cohen-Macaulay rings: solution to a problem of Grothendieck. J. Algebra, 252(1) (2002), 65-73.

[BK2] Bouchiba, S.; Kabbaj, S. Regularity of tensor products of k-algebras. Math. Scand. 115(1) (2014), 5-19.

[BH] Bruns,W.; Herzog, J. Cohen-Macaulay rings. Cambridge Stud. Adv. Math., 39. Cambridge University Press, Cambridge, 1993.

[Bo] Bourbaki, N. Algebra I. Chapters 1-3. Springer-Verlag, Berlin, 1998.

[EGA I] Grothendieck, A.; Dieudonné, J. Élements de géométrie algébrique, I. Grundlehren Math. Wiss., 166. Springer-Verlag, Berlin, 1971.

[EGA IV] Grothendieck, A.; Dieudonné, J. Éléments de géométrie algébrique, IV. Inst. Hautes Études Sci. Publ. Math., 20 (1964), 24 (1965), 28 (1966), 32 (1967).

[FR] Franco, L.; Rodicio, A.G. On the vanishing of the second Andre-Quillen homology of a local homomorphism. J. Algebra, 155(1) (1993), 137-141.

[FT] Foxby, H.B.; Thorup, A. Minimal injective resolutions under flat base change. Proc. Amer. Math. Soc. 67(1) (1977), 27-31.

[K] Kunz, E. Almost complete intersections are not Gorenstein rings. J. Algebra 28 (1974), 111-115.

[KK] Kiehl, R.; Kunz, E. Vollständige durchschnitte und p-basen. Arch. Math., 16 (1965), 348-362.

[M] Majadas, J. On tensor products of complete intersections. Bull. Lond. Math. Soc., 45(6) (2013), 1281-1284.

[MR] Majadas, J.; Rodicio, A.G. Smoothness, regularity and complete intersection. London Math. Soc. Lecture Note Ser., 373. Cambridge University Press, Cambridge, 2010.

[Mt] Matsumura, H. Commutative ring Theory. Cambridge Stud. Adv. Math., 8. Cambridge University Press, Cambridge, 1989.

[Mu] Mukhamedov, V.G. The behaviour of the Krull dimension of a commutative algebra under extension of the ground field. Russian Math. Surveys, 33(6) (1978), 247-248.

[R] Roberts, P. Rings of type 1 are Gorenstein. Bull. London Math. Soc., 15(1) (1983), 48-50.

[Sh1] Sharp, R.Y. Simplifications in the theory of tensor products of field extensions. J. London Math. Soc. (2), 15(1) (1977), 48-50.

[Sh2] Sharp, R.Y. The dimension of the tensor product of two field extensions. Bull. London Math. Soc., 9(1) (1977), 42-48.

[TY] Tousi, M.; Yassemi, S. Tensor products of some special rings. J. Algebra, 268(2) (2003), 672-676.

[WITO] Watanabe, K.; Ishikawa, T.; Tachibana, S.; Otsuka, K. On tensor products of Gorenstein rings. J. Math. Kyoto Univ., 9(3) (1969), 413-423.

Department of Mathematics, Faculty of Sciences, University Moulay Ismail, Meknes, Morocco E-mail address: bouchibasamir@gmail.com

Departamento de Álxebra, Facultade de Matemáticas, Universidade de Santiago de Compostela, E-15782 Santiago de Compostela, Spain

E-mail address: jesus.conde@usc.es

Departamento de Álgebra, Facultad de Matemáticas, Universidad de Santiago de Compostela, E-15782 Santiago de Compostela, Spain

E-mail address: j.majadas@usc.es 